\title{
Radio-interferometric Monitoring of FRB 131104: A Coincident AGN Flare, but No Evidence for a Cosmic Fireball
}

\author{
R. M. Shannon ${ }^{1,2,4}$ and V. Ravi ${ }^{3,4}$ \\ ${ }^{1}$ CSIRO Astronomy and Space Science, Australia Telescope National Facility, Box 76, Epping, NSW 1710, Australia; ryan.shannon@csiro.au \\ ${ }^{2}$ International Centre for Radio Astronomy Research, Curtin University, Bentley, WA 6102, Australia \\ ${ }^{3}$ Cahill Center for Astronomy and Astrophysics, California Institute of Technology, MC 249-17, Pasadena, CA 91125, USA; vikram@caltech.edu \\ Received 2016 November 17; revised 2017 February 17; accepted 2017 February 23; published 2017 March 8
}

\begin{abstract}
The localization of fast radio bursts (FRBs) has been hindered by the poor angular resolution of the detection observations and inconclusive identification of transient or variable counterparts. Recently a $\gamma$-ray pulse of $380 \mathrm{~s}$ duration has been associated with FRB 131104. We report on radio-continuum imaging observations of the original localization region of the FRB, beginning three days after the event and comprising 25 epochs over 2.5 years. We argue that the probability of an association between the FRB and the $\gamma$-ray transient has been overestimated. We provide upper limits on radio afterglow emission that would be predicted if the $\gamma$-ray transient was associated with an energetic $\gamma$-ray burst. We further report the discovery of an unusual variable radio source spatially and temporally coincident with FRB 131104, but not spatially coincident with the $\gamma$-ray event. The radio variable flares by a factor of 3 above its long-term average within 10 day of the FRB at $7.5 \mathrm{GHz}$, with a factor-of-2 increase at $5.5 \mathrm{GHz}$. Since the flare, the variable has persisted with only modest modulation and never approached the flux density observed in the days after the FRB. We identify an optical counterpart to the variable. Optical and infrared photometry, and deep optical spectroscopy, suggest that the object is a narrow-line radio active galactic nucleus.
\end{abstract}

Key words: black hole physics - galaxies: active - gamma rays: general - radio continuum: general

\section{Introduction}

Fast radio bursts (FRBs) represent a new phenomenological class of astrophysical transient. They are bright ( $\gtrsim$ Jy peak flux density) pulses of radio emission that show the effects of propagating though large column densities of plasma: dispersion through ionized plasma, multipath propagation due to inhomogeneities in the plasma (Lorimer et al. 2007; Thornton et al. 2013), and Faraday rotation due to magnetization of the plasma (Masui et al. 2015). The column densities exceed predictions for the Galaxy, suggesting that the FRBs are extragalactic and possibly cosmological in origin (Lorimer et al. 2007; Thornton et al. 2013; Ravi et al. 2016). They have hitherto only been detected using single-dish telescopes, which have poor angular resolution.

Only one FRB has been found to repeat (Spitler et al. 2016), which enables follow-up radio-interferometric campaigns to directly localize the source of the bursts. Direct radiointerferometric localization of the bursts with the Very Large Array and European VLBI Network (Chatterjee et al. 2017; Marcote et al. 2017) have identified a coincident radio continuum host, located in a dwarf galaxy at a redshift of $z \approx 0.2$ (Tendulkar et al. 2017). The nature of the the radio continuum source is unclear, with indications that it may be either a plerion powered by a young neutron star, an optically quiet active galactic nucleus (AGN), or sub-threshold pulses from the FRB itself.

For the remaining FRBs, which have not repeated, attempts at localization have relied on detecting counterpart multiwavelength transients that might be expected if the FRBs arise from cataclysmic explosions or outbursts. A claimed association of FRB 150418 with a centimeter-wavelength afterglow

\footnotetext{
${ }^{4}$ The authors contributed equally to this work.
}

and host galaxy (Keane et al. 2016) has been disputed and instead attributed to common AGN variability, either intrinsic (Vedantham et al. 2017; Williams \& Berger 2016) or caused by Milky Way scintillation (Akiyama \& Johnson 2016; Johnston et al. 2016). With so little detail on the locations of FRBs, theories for their production and sources are understandably varied, ranging from ultabright pulses from pulsars (Cordes \& Wasserman 2016), flares from magnetars (Lyubarsky 2014; Katz 2016), binary neutron star mergers (Wang et al. 2016), or cosmic strings (Cai et al. 2012).

We detected FRB 131104 (Ravi et al. 2015) with the $64 \mathrm{~m}$ Parkes radio telescope in the direction of the Carina dwarf spheroidal galaxy (Car dSph), $100 \mathrm{kpc}$ distant from Earth. The FRB has an electron column density, measured in units of dispersion measure (DM), of $779.0 \pm 0.2 \mathrm{pc} \mathrm{cm}^{-3}$ and shows evidence for temporal broadening associated with multipath propagation. Despite its detection in a targeted observation of the Car dSph, we have no evidence to associate the FRB with that galaxy. The FRB has not repeated in $\approx 100 \mathrm{hr}$ of follow-up observations at Parkes.

A $\gamma$-ray transient, Swift J0644.5-5111, has recently been associated with the FRB at the $3.2 \sigma$ to $3.4 \sigma$ confidence level (DeLaunay et al. 2016). The emission was detected in an offaxis position with the Swift satellite's Burst Alert Telescope (BAT; Barthelmy et al. 2005), coincident with the FRB in position and time. The transient duration was $\sim 380 \mathrm{~s}$, with an inferred energy output of $5 \times 10^{51} \mathrm{erg}$. DeLaunay et al. (2016) suggested that the $\gamma$-ray emission (assumed to be associated with this FRB) was generated by shocked relativistic plasma in a cosmological explosion, or in an accretion episode associated with a supermassive black hole. We discuss the claimed association between Swift J0644.5-5111 and FRB 131104 in Section 2, addressing specifically the mismatch (noted by 
DeLaunay et al.) between the low rate of such transients observed by Swift/BAT and the high FRB all-sky rate.

Here, we report on a centimeter-wavelength radio monitoring campaign of the Parkes localization region of FRB 131104 and the discovery of an unusual, variable radio source (AT J0642.9-5118) that flares coincident in time and location with FRB 131104. AT J0642.9-5118 is not coincident with Swift J0644.5-5111; indeed, our observations exclude any bright radio afterglow of Swift J0644.5-5111. In Section 3, we present radio observations of the field and the light curve of AT J0642.9-5118. In Section 4, we present an optical characterization of AT J0642.9-5118. We discuss the implications of our observations in Section 5 and conclude the Letter in Section 6.

\section{The $\gamma$-Ray Transient Coincident with FRB 131104}

Swift J0644.5-5111 was discovered within the $15^{\prime}$ diameter half-power circle of the beam (\#5) of the Parkes $21 \mathrm{~cm}$ multibeam receiver in which FRB 131104 was detected, 6.2 from the beam center. Its position at the edge of the BAT field of view, illuminating only $2.9 \%$ of detectors, resulted in a $4.2 \sigma$ detection in the image plane despite its high fluence of $4 \times 10^{-6} \mathrm{erg} \mathrm{cm}^{-2}$. Assuming a distance of $3.2 \mathrm{Gpc}$ for FRB 131104 based on comparing its extragalactic DM with models for the ionized content of the universe, the isotropic energy output of Swift J0644.5-5111 was $5 \times 10^{51} \mathrm{erg}$, with a duration of $\sim 380 \mathrm{~s}$. This is somewhat longer, and somewhat less energetic, than most long-duration gamma-ray bursts (GRBs) detected by Swift (Gehrels et al. 2009), but is inconsistent with other GRB subtypes (e.g., ultra-long or short GRBs). Murase et al. (2017) consider it likely that, largely independent of the source model, a radio afterglow would have been present. We constrain such an afterglow in Section 5.2 using our observations.

DeLaunay et al. (2016) quote a false-alarm probability of observing an event such as Swift J0644.5-5111 spatially and temporally coincident with FRB 131104 between $3.2 \sigma$ and $3.4 \sigma$. These quantities were derived by estimating the probability of observing an event as bright as, or brighter than, Swift J0644.5-5111 within an arbitrary $15^{\prime}$ diameter region of the BAT field of view at similar coding percentages and an arbitrary $300 \mathrm{~s}$ interval. These false-alarm probabilities do not readily translate into significances for the association between the FRB and the Swift event. Because there is no clear hypothesis of what type of $\gamma$-ray transient would be associated with an FRB, the probability of a false detection is underestimated as the team has chosen to stop their search when they achieved a positive result.

A good example of this problem is the initial detection of the FRB, which was detected within 90 minutes of a survey for FRBs from Milky Way dwarf satellites. If we had chosen to stop our search at this stage, the false-alarm probability would have led us to conclude that FRBs are associated with Milky Way satellites. However, despite the improbability of our finding (odds ratio of approx. 400:1, assuming an FRB detection with a fluence $\geqslant$ that of FRB 131104 every 25 days at Parkes), extensive follow-up observations, and observations of other FRBs do not offer any further evidence to support this hypothesis.

Thus, we argue that the false-alarm rates quoted by DeLaunay et al. (2016) for the observation of Swift J0644.5 -5111 cannot be directly interpreted as statistical evidence for an association with FRB 131104. The situation would be different if we knew a priori that FRBs are associated with events such as Swift J0644.5-5111, in which case the falsealarm rates could be used to reject the null hypothesis of no association specifically between Swift J0644.5-5111 and FRB 131104. The case for an association would also be stronger if the data motivated a physical model linking the two events. Consider, for example, a scenario where an associated $\mathrm{X}$-ray transient had been detected and localized by Swift, and if follow-up multiwavelength observations had revealed an unusual type of occurrence that could be associated with an FRB, such as binary neutron star merger.

Furthermore, following an argument made by Vedantham et al. (2016b), we argue that it is unlikely that all FRBs could be associated with events like Swift J0644.5-5111. This is because of the inconsistency between the inferred all-sky $\gamma$-ray pulse rate and the FRB rate, as noted by DeLaunay et al. (2016). If Swift J0644.5-5111 had occurred in the region of the BAT field of view with $>90 \%$ coding, it would have resulted in an image-based burst trigger. DeLaunay et al. (2016) estimate that the rate of long-duration image-triggered events, presumably similar to Swift J0644.5-5111, is $25 \mathrm{yr}^{-1}$. We make the conservative assumption that these events all have FRB counterparts, regardless of their fluence or classification. The $100 \%$ coding region of BAT is $\approx 1000 \mathrm{deg}^{2}$ (Barthelmy et al. 2005), which we (conservatively) equate with the $>90 \%$ coding region. In this region, we predict that BAT should have been sensitive to the counterparts of between 8800 and 17,600 FRBs in a year. We calculate this using the fluencecomplete FRB rate of $2500 \mathrm{sky}^{-1}$ day $^{-1}$ events with fluences $>2$ Jy ms (Keane \& Petroff 2015), and assume both that the FRB source counts are consistent with a Euclidean universe and that Swift obtains a $>50 \%$ observing duty cycle. Therefore, from the ratio of the $\gamma$-ray event rate to the radio event rate, only between $1 / 350$ and $1 / 700$ FRBs could be expected to have long-duration $\gamma$-ray counterparts such as Swift J0644.5 -5111. If FRB 131104 was indeed associated with Swift J0644.5-5111, it would have to be of a fundamentally different, much rarer class than the other FRBs.

\section{Radio-interferometric Observations}

We commenced monitoring the field of FRB 131104 with the Australia Telescope Compact Array (ATCA) three days after the FRB was detected at Parkes. Our observations were conducted over 25 epochs spanning 2.5 years. Visibilities were computed using the Compact Array Broadband Backend (Wilson et al. 2011) over two $2 \mathrm{GHz}$ width tunable bands, centered at 5.5 and $7.5 \mathrm{GHz}$. A 42-pointing mosaic was necessary to cover to twice the half-power beam point of Parkes observations (which is the first null in the primary beam) at the highest frequency of the ATCA observations. This was especially crucial because of the possibility of a population of ultrabright FRBs that could be detected in the outer main beam or sidelobe of the telescope (Vedantham et al. 2016a).

Observations were conducted in a variety of array configurations, with maximum baseline lengths varying between $214 \mathrm{~m}$ and $6 \mathrm{~km}$. Usually six antennas were available, but some observations were conducted with five antennas (particularly in the lower-resolution arrays where inclusion of a sixth very distant antenna complicates imaging) and one with four. The lower spatial resolution observations suffered from higher noise, but other problems such as source confusion were not a 
problem because the field is relatively sparse. Data were bandpass calibrated using observations of either PKS 0823 -500 or PKS $1934-638$, and flux calibrated using the latter. Phase calibration was conducted with regular observations of the unresolved radio galaxy J0625-5438. Data were reduced using the miriad data reduction package (Sault et al. 1995). The visibilities for each pointing were imaged and deconvolved independently (using multi-frequency synthesis and cleaning) and then combined to form a composite image. Noise levels were typically $30 \mu \mathrm{Jy}_{\text {beam }}{ }^{-1}$ in the mosaicked observations. We investigated the role of self-calibration (both phase-only and amplitude-and-phase self-calibration) on our flux-density measurements. We found that while self-calibration improved image fidelity it did not significantly alter flux-density measurements. ${ }^{5}$

Figure 1 shows composite images formed from the $5.5 \mathrm{GHz}$ (top) and $7.5 \mathrm{GHz}$ (bottom) observations of the field. The rms noise levels in the two images are, respectively, 14 and $19 \mu \mathrm{Jy}_{\text {beam }}{ }^{-1}$. The width of the primary beam of Parkes, to the first null, is shown as the blue circle. The $90 \%$ containment region for Swift J0644.5-5111 is shown as the red circle. There are no sources within this region in either the mosaics of all our data shown in Figure 1 or in individual epochs, allowing us to place $5 \sigma$ limits on persistent sources at $5.5 \mathrm{GHz}$ and $7.5 \mathrm{GHz}$ of 70 and $100 \mu \mathrm{Jy}$, respectively. In Table 1, we show rms noise levels $(1 \sigma)$ in the region coincident with the ATCA transient, for observations that covered this region.

Within the field of view, we have identified a strongly variable source, which we refer to as AT J0642.9-5118. The location of the source on the sky is (J2000) $\alpha=6^{\mathrm{h}} 42^{\mathrm{m}} 57^{\mathrm{s}} .154(3), \delta=-51^{\circ} 18^{\prime} 17^{\prime \prime}$.70(7). The light curve for the source is presented in Figure 2. In the week after the occurrence of FRB 131104, the source brightens by a factor of 2 , exceeding $1200 \mu \mathrm{Jy}$ in the $7.5 \mathrm{GHz}$ band. During the brightening, the spectrum also inverts. Other sources in the field do not show this level of variability, suggesting that miscalibration has not introduced the flux variation. We verified that this source, so far from beam center, could represent the FRB counterpart by observing the bright pulsar J1456-6843 at the position of the variable source in the Parkes focal plane. The pulsar was only detected in beam \#5 of the multibeam. The pulsar observation had a higher signal-to-noise ratio than the FRB, indicating that a single-beam detection of the FRB is consistent with the burst originating from the position of the ATCA variable source.

After identifying AT J0642.9-5118, we conducted more sensitive single-pointing observations at $2.1,5.5$, and $7.5 \mathrm{GHz}$. Observation and data reduction in the $2.1 \mathrm{GHz}$ band followed the same procedures as in the mosaicked observations. In the $2.1 \mathrm{GHz}$ band, the major differences were that only $2 \mathrm{GHz}$ of bandwidth was available, and phase calibration used the radio galaxy PKS 0647-475. For these targeted observations, image rms noise was typically $30 \mu \mathrm{Jy} \mathrm{beam}^{-1}$ in the $2.1 \mathrm{GHz}$ band, and $10 \mu \mathrm{Jy}_{\text {beam }^{-1}}$ in the higher-frequency images. We note that these targeted observations do not cover the containment region of the $\gamma$-ray pulse.

There was a modest re-brightening of AT J0642.9-5118 approximately 300 days after the initial flare. After the initial flare, the flux density at $7.5 \mathrm{GHz}$ has a mean value of $395 \mu \mathrm{Jy}$

\footnotetext{
5 Johnston et al. (2017) noted $\mathrm{a} \approx 10 \%$ downward bias in flux-density measurements in mosaicked data sets. We found that this was mitigated by imaging with the source of interest at the reference pixel of the image.
}
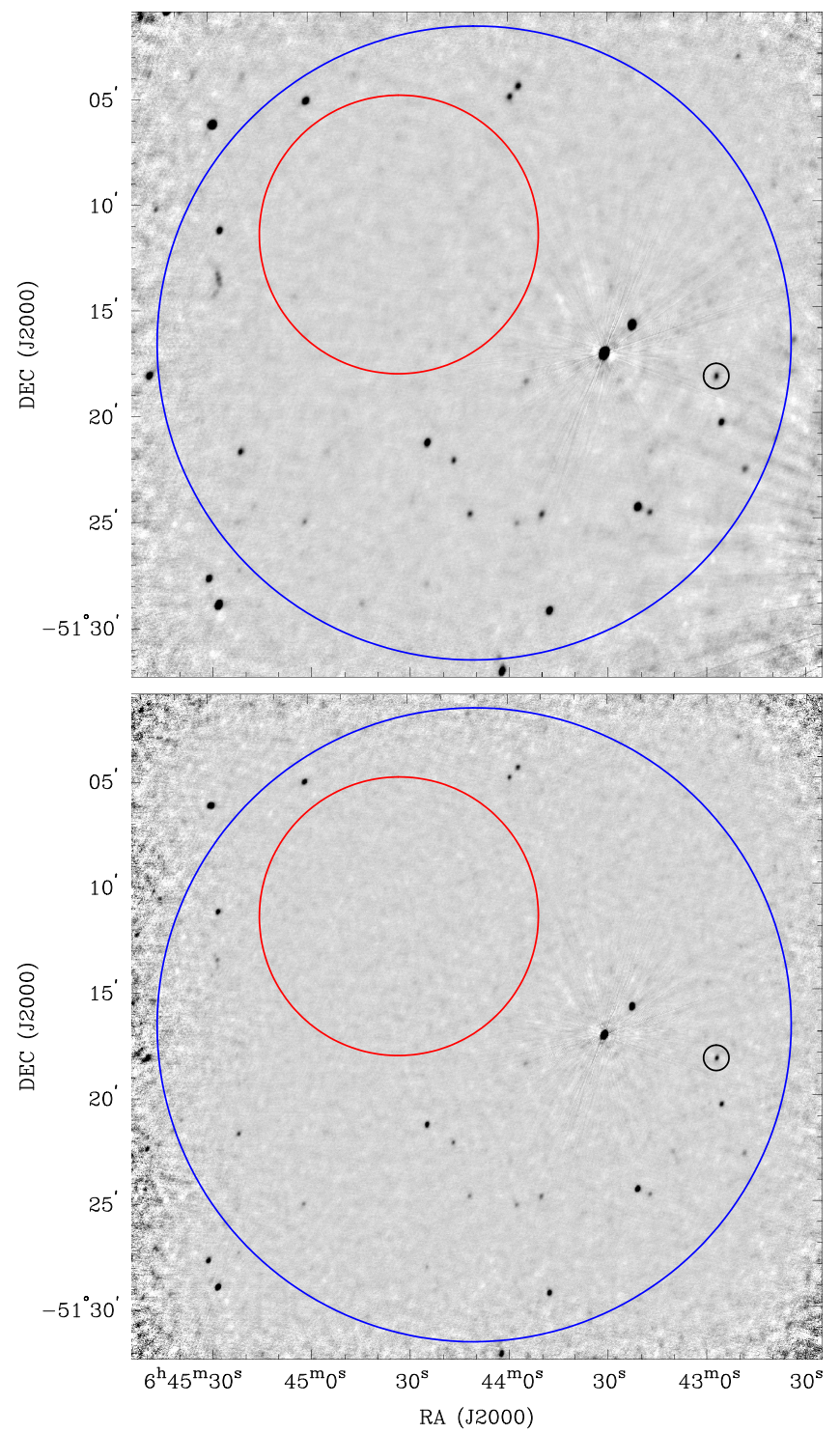

Figure 1. Radio continuum images of the field surrounding FRB 131104 in the 5.5 GHz band (top) and $7.5 \mathrm{GHz}$ band (bottom). The blue circle shows the beam of Parkes telescope (to twice the half-power point, which is approximately the first null in the beam pattern). The 5.5 and $7.5 \mathrm{GHz}$ image rms flux densities within the blue circle are $15 \mu \mathrm{Jy}_{\text {beam }}^{-1}$ and $20 \mu \mathrm{Jy}_{\text {beam }^{-1}}$, respectively. The red circle shows the $90 \%$ confidence region for the Swift transient. The black circle shows the position of the unusual variable AT J0642.9-5118. In both plots, the grayscale ranges linearly from -100 to $500 \mu \mathrm{Jy}_{\text {beam }^{-1}}$.

and an rms value of $80 \mu \mathrm{Jy}$, suggesting that the $1.2 \mathrm{mJy}$ event is a $10 \sigma$ event temporally coincident with the $\mathrm{FRB}$; at $5.5 \mathrm{GHz}$, the mean flux density was $390 \mu \mathrm{Jy}$ with an rms value $100 \mu \mathrm{Jy}$ after the flare. The modulation index in the $5.5 \mathrm{GHz}(7.5 \mathrm{GHz})$ band is $0.3(0.2)$ when excluding the first three observations and $0.4(0.5)$ when including them. Measured flux densities for the variable radio source are shown in Table 1.

\section{Optical Observations of AT J0642.9-5118}

\subsection{Imaging}

Following the identification of AT J0642.9-5118, we obtained images of its optical counterpart with the Gemini Multi-Object Spectrograph (GMOS; Hook et al. 2004) at the Gemini-South telescope (project ID GS-2015A-Q-37). Our 
Table 1

ATCA Flux Density Measurements

\begin{tabular}{|c|c|c|c|c|c|c|c|}
\hline $\begin{array}{l}\text { Date } \\
\text { MJD }\end{array}$ & $\underset{\text { (day) }}{\Delta t}$ & Array & $\begin{array}{c}S_{\mathrm{AGN}, 5.5} \\
(\mu \mathrm{Jy})\end{array}$ & $\begin{array}{c}S_{\mathrm{AGN}, 7.5} \\
(\mu \mathrm{Jy})\end{array}$ & $\begin{array}{c}S_{\mathrm{AGN}, 2.1} \\
(\mu \mathrm{Jy})\end{array}$ & $\begin{array}{l}S_{\gamma, 5.5} \\
(\mu \mathrm{Jy})\end{array}$ & $\begin{array}{l}S_{\gamma, 7.5} \\
(\mu \mathrm{Jy})\end{array}$ \\
\hline 56603 & 3 & $6 \mathrm{~B}$ & $820(90)$ & $680(100)$ & $\ldots$ & 43 & 36 \\
\hline 56609 & 9 & $6 \mathrm{~B}$ & $950(100)$ & $1220(100)$ & $\ldots$ & 23 & 29 \\
\hline 56675 & 75 & $6 \mathrm{~A}$ & $480(50)$ & $410(70)$ & $\ldots$ & 30 & 50 \\
\hline 56912 & 312 & $6 \mathrm{~B}$ & $560(80)$ & $350(80)$ & $\ldots$ & 32 & 43 \\
\hline 56915 & 315 & $\mathrm{H} 214$ & $680(70)$ & $390(120)$ & $\ldots$ & 84 & 63 \\
\hline 57009 & 409 & $6 \mathrm{~A}$ & $420(20)$ & $400(20)$ & $390(50)$ & $\ldots$ & $\ldots$ \\
\hline 57051 & 451 & $6 \mathrm{~A}$ & $350(10)$ & $320(10)$ & $\ldots$ & $\ldots$ & $\ldots$ \\
\hline 57081 & 481 & $6 \mathrm{C}$ & $220(30)$ & $280(30)$ & $230(20)$ & $\ldots$ & $\ldots$ \\
\hline 57099 & 499 & $\mathrm{H} 214$ & $210(20)$ & $350(20)$ & $\ldots$ & $\ldots$ & $\ldots$ \\
\hline 57159 & 559 & $1.5 \mathrm{C}$ & $230(40)$ & 290(70) & $\ldots$ & 25 & 28 \\
\hline 57327 & 727 & $6 \mathrm{~A}$ & $350(10)$ & $300(20)$ & $240(40)$ & $\ldots$ & $\ldots$ \\
\hline 57402 & 802 & $750 \mathrm{C}$ & $330(20)$ & $390(10)$ & $400(80)$ & $\ldots$ & $\ldots$ \\
\hline 57406 & 806 & $750 \mathrm{C}$ & $320(20)$ & $310(20)$ & $280(40)$ & $\ldots$ & $\ldots$ \\
\hline 57438 & 838 & $6 \mathrm{~B}$ & $430(20)$ & $390(20)$ & $330(30)$ & $\ldots$ & $\ldots$ \\
\hline 57439 & 839 & $6 \mathrm{~B}$ & $420(20)$ & $450(20)$ & $330(40)$ & $\ldots$ & $\ldots$ \\
\hline 57441 & 841 & $6 \mathrm{~B}$ & $440(20)$ & $430(20)$ & $440(40)$ & $\ldots$ & $\ldots$ \\
\hline 54446 & 846 & $6 \mathrm{~B}$ & $380(20)$ & $350(20)$ & $480(60)$ & $\ldots$ & $\ldots$ \\
\hline 57456 & 856 & $6 \mathrm{~B}$ & $360(20)$ & $330(20)$ & $340(40)$ & $\ldots$ & $\ldots$ \\
\hline
\end{tabular}

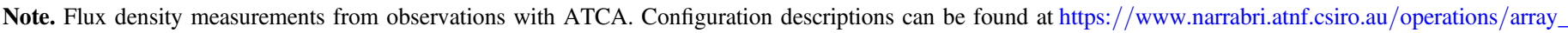
configurations/configurations.html. The columns $S_{\gamma}$ show the $1-\sigma$ rms noise levels of the image in the area coincident with the $\gamma$-ray pulse.

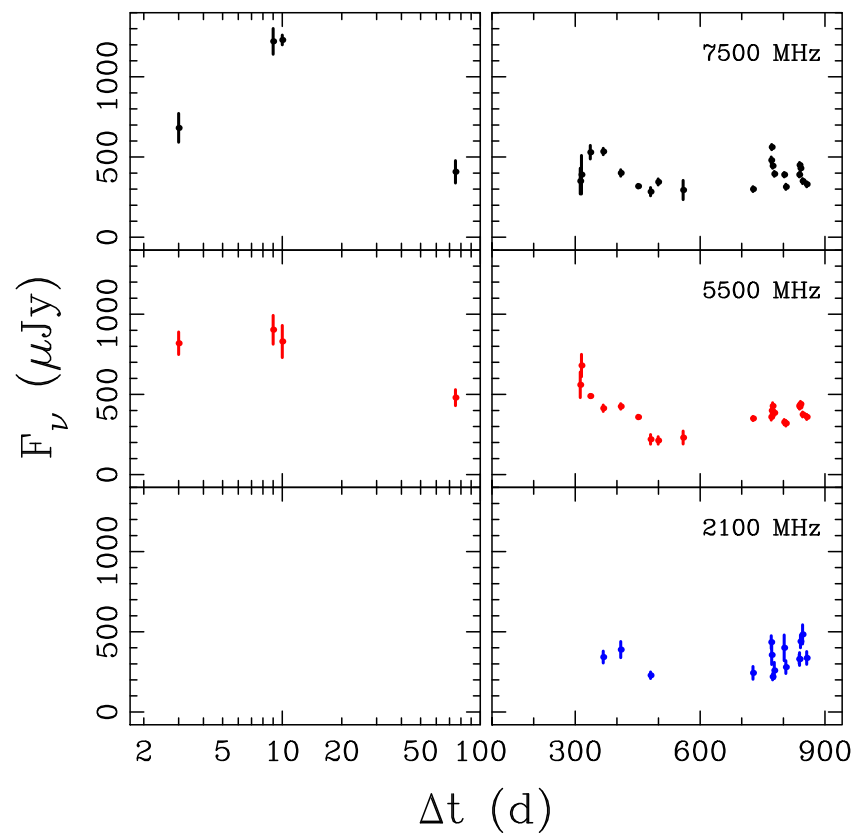

Figure 2. Radio light curve for the variable radio source AT J0642.9-5118 From the bottom to top panels, we show the flux density measured in the bands centered at 2.1, 5.5, and 7.5 GHz. The $x$-axis is the time since FRB 131104. For $\Delta t<100$ day, we show the light curve on a logarithmic time axis (left panels). For $\Delta t>100$ day, the axis is linear (right panels).

observations were conducted on MJD 57141 in the $g^{\prime}$ and $r^{\prime}$ bands using the Hamamatsu CCDs (Gimeno et al. 2016), under photometric conditions with 0 ". 6 FWHM seeing. Four dithered exposures were taken in each band, totaling $2617 \mathrm{~s}$ in the $g^{\prime}$-band and $2322 \mathrm{~s}$ in the $r^{\prime}$-band. We reduced the data using the standard GMOS pipeline tasks in the Gemini IRAF package. We used facility bias and flat-field exposures nearest in time to our observations to correct the data, and co-added all images following subtraction of dithering offsets. Astrometric corrections were applied to the images using D. Perley's autoastrometry software ${ }^{6}$, using the USNO B1.0 catalog as a reference (Monet et al. 2003), with 0."32 accuracy.

We identified a point-like counterpart to the radio source that is the north-west component of a close ( 0 ." 6 separation) double (Figure 3, left and middle panels). We term this source G1, and its southeastern companion G2. As we did not observe a photometric standard field, we used the GMOS-South photometric equation defined online ${ }^{7}$ to set the flux scale. We modeled the point-spread function using nearby stars and used this to model G1 and G2 as two point sources, finding a satisfactory fit to the observation and noise-like residuals after subtracting the two point sources. For G1, we obtained AB magnitudes of $g^{\prime}=22.82 \pm 0.02$ and $r^{\prime}=22.51 \pm 0.02$, and for G2 we obtained $g^{\prime}=22.87 \pm 0.02$ and $r^{\prime}=21.77 \pm 0.01$. At this position, the Galactic extinction is $0.208 \mathrm{mag}$ in the $g^{\prime}$ band, and 0.144 mag in the $r^{\prime}$ band (Schlafly \& Finkbeiner 2011).

We also obtained imaging observations in the $J$ band with the FourStar instrument (Persson et al. 2013) on the Magellan-Baade telescope at Las Campanas Observatory. The observations, conducted on MJD 57270 under photometric conditions with 0." 65 FWHM seeing, were split into 18 dithered exposures totaling $1153 \mathrm{~s}$. The data were reduced using the standard

\footnotetext{
6 http://www.astro.caltech.edu/ dperley/programs/autoastrometry.py

7 https://www.gemini.edu/sciops/instruments/gmos/calibration/ photometric-stds
} 

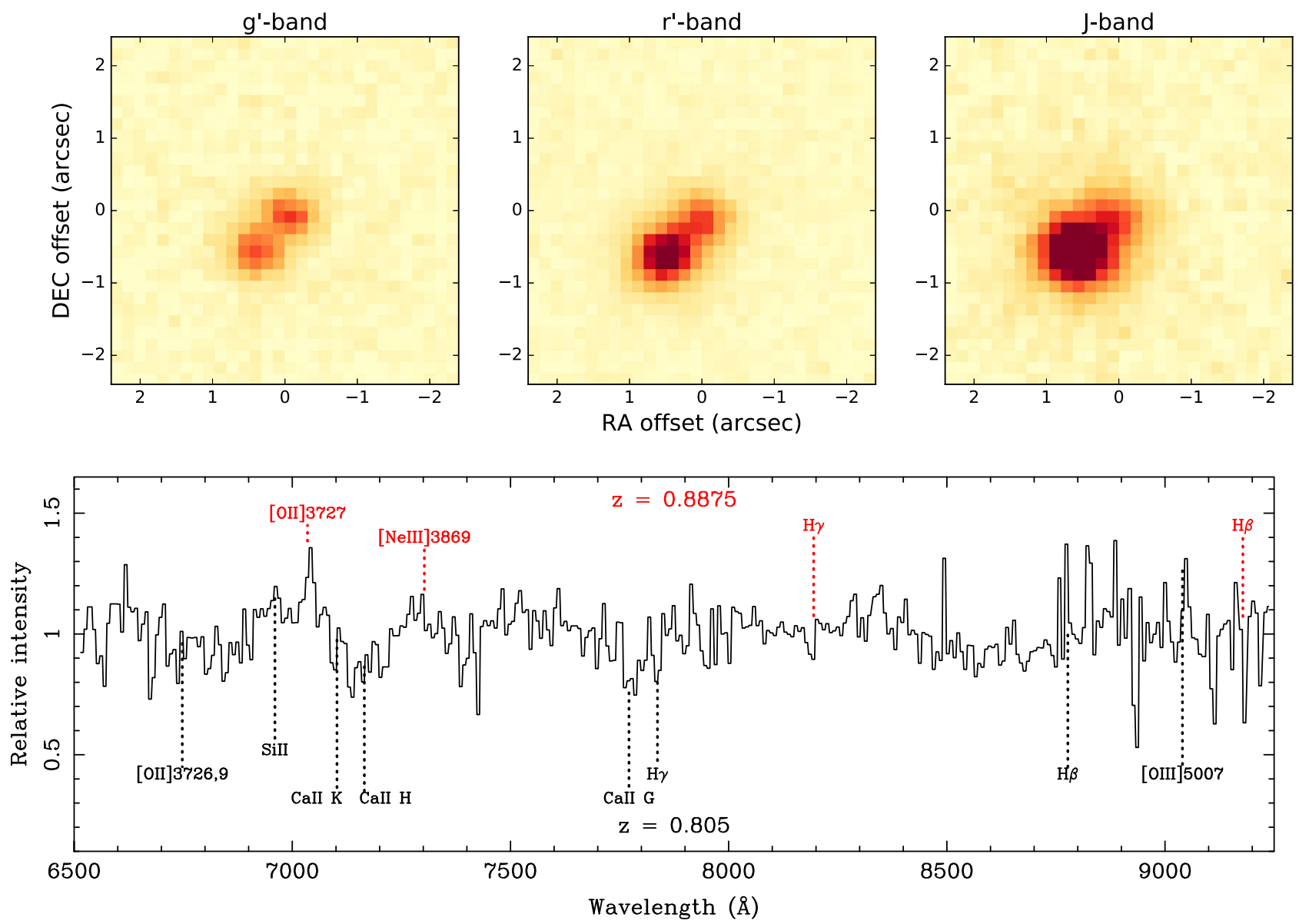

Figure 3. Top: images of optical counterpart and its pair in $g^{\prime}, r^{\prime}$ (GMOS-South), and $J$ bands (Magellan). Source G1, which is the counterpart to AT J0642.9-5118, is in the image center, and G2 is to the lower left (southeast). The intensity scaling (zero-point) is common between the images. Bottom: continuum-divided combined spectrum obtained of the pair of optical sources. We show features potentially associated with source G1 in black and source G2 in red, with the corresponding redshifts quoted in the figure. Note that numerous artifacts due to imperfect sky-line subtraction are present. The instrumental FWHM of the spectrograph was $3 \AA$.

FourStar pipeline. We calibrated the photometry and astrometry of the image using 2MASS point sources (Skrutskie et al. 2006), attaining 0.2 astrometric accuracy. The resulting detections of G1 and G2 are shown in the right panel of Figure 3. Using the same technique as above, we measure $\mathrm{AB}$ magnitudes of $J=21.54 \pm 0.03$ for $\mathrm{G} 1$, and $J=20.24 \pm 0.01$ for G2. The Galactic extinction in the $J$ band is $0.045 \mathrm{mag}$ (Schlafly \& Finkbeiner 2011).

The point-source catalog of the Widefield Infrared Survey Explorer (WISE; Wright et al. 2010) contains a source, WISE J064257.16-511817.8, which is coincident with G1 and is detected in the two shortest wavelength bands. Its $(\mathrm{AB})$ magnitudes are $W 1=17.9 \pm 0.1$ and $W 2=16.7 \pm 0.2$. Based on this color, the source is consistent with an AGN (Stern et al. 2012). The optical colors are also consistent with an AGN at moderate redshift, such that $\mathrm{Ly} \alpha$ is blueward of our observations (Smolčić et al. 2006). While the angular resolution of WISE ( $>6$ arcsec) is much lower than the radiointerferometric and Gemini observations, the astrometry is sufficiently accurate (uncertainty $\approx 0.4 \mathrm{arcsec}$ ) to associate WISE with the AT J0642.9-5118 and its optical counterpart G1, rather than source G2, or a blend of both.

\subsection{Spectroscopy}

We also obtained optical spectra of G1 and G2 using GMOS at Gemini-South (Project ID GS-2015B-Q-24). We used a 1" longslit oriented along the axis of G1 and G2 (position angle of $317^{\circ}$ ). Our observations in the red part of the spectrum were conducted on MJD 57362 using the R400 grating with the GG455 order-blocking filter. Four $920 \mathrm{~s}$ exposures were taken at a mean airmass of 1.2 , with two centered on $8610 \AA$ and two centered on $8510 \AA$ to cover the gaps between CCDs. Our blue observations were conducted on MJD 57367 at a mean airmass of 1.1 using the B600 grating with no filter, and three $920 \mathrm{~s}$ exposures (two centered on $5060 \AA$, and one centered on $4960 \AA$ ). We reduced the data using the standard GMOS pipeline, with a bias observation obtained on MJD 57363, and flat-field observations taken in between our science exposures.

Unfortunately, intermittent high cirrus was present, precluding accurate spectrophotometric calibration and making sky emission lines difficult to subtract. We nonetheless used observations of a spectrophotometric calibrator on MJD 57562 (LTT 3218) to calibrate telluric absorption features. As the seeing on both nights was $\sim 1^{\prime \prime}$ FWHM, as measured from acquisition images, we could not deconvolve the spectral traces of G1 and G2. Furthermore, some light from G1 was likely refracted out of the slit as GMOS does not contain an atmospheric dispersion corrector. Hence, following optimal extraction of the spectra, we only considered data taken using the R400 grating at wavelengths shorter than $9250 \AA$ and divided the data by a smooth polynomial fit to the continuum. The resulting spectrum, which contains numerous artifacts due 
to imperfect sky subtraction and is binned to $8 \AA$ resolution, is shown in Figure 3.

We tentatively identify two redshifted systems in this spectrum: one at $z=0.805 \pm 0.001$ and one at $z=0.8875 \pm 0.001$. The first system is consistent with the spectra of early-type galaxies (Polletta et al. 2007), a hypothesis which is additionally evidenced by a possible spectral break in the continuum around $4000 \AA$. We thus interpret it as corresponding to G2. The redshift of the second system, presumably G1 (the radio source), is estimated primarily using the strong emission line at $7040 \AA$ and by assuming (based on the WISE and blue optical colors) that it is an AGN. Identifying the $7040 \AA$ line with [O II]3272 $\AA$ results in a clear prediction, specifically that strong emission lines (e.g., Ly $\alpha, \mathrm{C}$ IV , Mg II) should be seen at shorter wavelengths. It appears to exhibit $\mathrm{H} \gamma$ and $\mathrm{H} \beta$ in absorption. The lack of these normally broad lines in emission, combined with the compact nature of its radio counterpart evidenced by the variability, is suggestive of a narrow-line radio galaxy (Osterbrock 1978), or perhaps a radio-loud narrow-line Seyfert 1 (Komossa 2008). More sensitive spectra with broader wavelength coverage would help in this classification, for example, by searching for the Fe II emission features that distinguish the narrow-line Seyfert 1 class.

\section{Discussion}

\subsection{Limits on Afterglows from the $\gamma$-Ray Pulse}

Our observations can be used to search for afterglow emission associated with the potential $\gamma$-ray transient Swift J0644.5-5111. In the classic fireball model (Mészáros \& Rees 1997; Frail et al. 2000), the flux density of radio synchrotron emission is directly related to the input energy. Murase et al. (2017) calculated the flux density assuming the spectrum is not self-absorbed and that the frequency of interest is below the peak of the spectrum so that the flux density is still rising. This is a reasonable assumption for our observations within 9 and 10 days after the FRB. Assuming a distance $D=3.3 \mathrm{Gpc}$ consistent with the pulses extragalactic DM, after time $T=10$ days at a frequency $\nu=5.5 \mathrm{GHz}$, the flux density is

$$
\begin{array}{rl}
S_{\nu}=4 & 70 \mu \mathrm{Jy}\left(\frac{\nu}{5.5 \mathrm{GHz}}\right)^{1 / 3}\left(\frac{E_{\gamma}}{10^{51.7} \mathrm{erg}}\right)^{5 / 6} \\
\times & \left(\frac{n}{0.1 \mathrm{~cm}^{-3}}\right)^{1 / 2}\left(\frac{\epsilon_{B}}{10^{-2}}\right)^{1 / 3}\left(\frac{\epsilon_{e}}{0.1}\right)^{-2 / 3} f_{e}^{5 / 3} \\
\times & g(2.4)^{-2 / 3}\left(\frac{T}{10 \text { day }}\right)^{1 / 2}\left(\frac{D}{3 \mathrm{Gpc}}\right)^{-2},
\end{array}
$$

where $E$ is the energy emitted in $\gamma$-rays, $n$ is the electron number-density of the shocked medium, $\epsilon_{B}$ is the magnetic energy fraction, $\epsilon_{e}$ is the nonthermal-electron energy density, $f_{e}$ is the nonthermal-electron energy injection fraction, $g(s)=(s-2) /(s-1)$, and $s$ is slope of an assumed power-law energy distribution for the electrons (Rees \& Mészáros 1998). This expression also assumes that there is $100 \%$ efficiency in converting kinematic energy in the explosion to $\gamma$-ray emission. For fiducial assumptions for these parameters, we could have detected the source in both the 5.5 and $7.5 \mathrm{GHz}$ observations 10 days after the explosion, with a significance of $12-20 \sigma$. This suggests that either the input luminosity is smaller than estimated in DeLaunay et al. (2016), that the environment surrounding the burst is unlike that of long $\gamma$-ray bursts or core-collapse supernova explosions, or that the $\gamma$-ray transient is unrelated to the FRB or spurious.

Even if the $\gamma$-ray pulse was cause by an energetic explosion, it is possible that radio afterglow self-absorbed after 10 days and fainter than would be estimated from Equation (1). Strong limits are placed on the radio emission coincident with the $\gamma$ ray pulse 300 days and 600 days after the emission (see Table 1), which constrain energetics of slower explosions, that, for example, remain synchrotron self-absorbed for longer durations. Finally, we note that it is possible that the $\gamma$-ray pulse is not associated with a classic explosion; in this case, radio afterglow emission would not be expected and our radio observations are unconstraining.

\subsection{The ATCA Variable Source}

We interpret the variable radio source AT J0642.9-5118 as emission from compact components in a radio-loud AGN. This is evidenced by the persistent radio variability on timescales of days to months, the optical to mid-infrared colors of its host system, and its possible spectral identification. The light curve of the flare following FRB 131104, with the spectrum inverting when it brightens, is consistent with the classic picture of an expanding and cooling synchrotron bubble. Although the flare light curve is consistent with the radio afterglows of relativistic transients (e.g., Frail et al. 2000), the persistence and low-level variability of the radio source beyond the flare means that we have no evidence to favor a transient coincident with a variable radio source, over simply a variable radio source.

AT J0642.9-5118 is nonetheless interesting. This object has substantial differences from the variable radio source identified with FRB 150418 (Johnston et al. 2017; Keane et al. 2016; Williams \& Berger 2016). First, we clearly identify the flare of AT J0642.9-5118 with the days immediately after the FRB, as we observe the flux density rise and the spectrum invert. Additionally, scintillation in the Milky Way interstellar medium is less likely to cause the variability of AT J0642.9 -5118 because the source is at a relatively high galactic latitude $\left(b=-22^{\circ}\right)$, diffractive and refractive scintillation are likely to cause rapid variability (Cordes \& Lazio 2002; Akiyama \& Johnson 2016). It is possible that the source was magnified by an extreme scattering event (Bannister et al. 2016), but that is improbable as only one in $\approx 2000$ compact sources are undergoing one at a given time.

Perhaps most importantly, AT J0642.9-5118 has not rebrightened to within a factor of two of its flux densities as the peak of the flare, unlike the case for FRB 150418 (Johnston et al. 2017; Williams \& Berger 2016). The flare of AT J0642.9 -5118 following FRB 131104 thus appears to be a transient occurrence within the scope of our monitoring of its flux density.

Blind surveys for transients at lower frequencies find objects with such extreme variability (factor of three on few-month timescales) only very rarely (e.g., Mooley et al. 2016). This is not surprising. Assuming constant brightness temperature, intrinsic AGN variability timescales scale with frequency 
proportional to $\approx \nu^{-1}$. Transient AGN flare events at higher frequencies are hence generally expected to be shorter in time, and are often also larger in modulation, than at lower frequencies (e.g., Hovatta et al. 2008). Scintillation timescales in the strong scintillation regime are expected to be more rapid. The post-FRB flare of AT J0642.9-5118 is clearly most dramatic at the highest observing frequency.

The temporal coincidence of AT J0642.9-5118 flare with FRB 131104 nonetheless motivates us to consider the possibility that it is associated with the FRB. In this case, AGN activity would be implicated in FRB production. The potential redshift $(z=0.8875)$ of AT J0642.9-5118 is consistent ${ }^{8}$ with the extragalactic DM of the FRB (Dolag et al. 2015). Determining the extragalactic contribution to the DM is complicated by the unknown host DM contribution, which has been thought to be both large $\left(>100 \mathrm{pc} \mathrm{cm}^{-3}\right.$; Masui et al. 2015) and small $\left(<30 \mathrm{pc} \mathrm{cm}^{-3}\right.$; Ravi et al. 2016; Chatterjee et al. 2017) for other other FRBs, and is unknown for FRB 131104. While other source channels have been more strongly advocated for FRBs, it is not implausible that AGNs could produce FRBs. Millisecondduration radio pulses propagating through relativistic plasma in AGN jets may be immune to both absorption and scattering effects (Lyubarsky 2008), implying that FRBs originating close to launching regions could be observed from AGN viewed along the jets. Mechanisms (e.g., Romero et al. 2016) have been proposed for the production of FRBs in AGN jets, analogous to the mechanisms for generating $\mathrm{TeV}$ photons.

There are, however, reasons to disfavor an association between AT J0642.9-5118 and the FRB. The background transient and variable event rate at $7.5 \mathrm{GHz}$ (where the flare is the most prominent), and hence the false-alarm rate for the association, is poorly constrained, as searches for transients and variables have focused on lower frequencies $(<6 \mathrm{GHz}$; Bell et al. 2015; Mooley et al. 2016) and lower Galactic latitudes (Ofek et al. 2011). Even so, the high all-sky FRB rate needs to be reconciled with the background variable rate (Vedantham et al. 2016b). Intrinsic AGN variability is likely to dominate the background slow-transient rate.

Further analysis of the radio AGN population and its variability properties in comparison with the FRB rate would be required to assess how commonly a single object would be expected to emit an FRB, and what its signature could be. Further physical modeling of the conditions and orientations under which FRBs could escape AGNs would help refine such an analysis. This analysis would be further aided by a large area survey for transient and variable sources at high frequencies, as well as dedicated follow-up observations of other FRBs, in particular to assess the frequency of short-duration flares in AGNs. However, a substantially more constraining result would be the direct interferometric localization of a population of FRBs to flaring AGN. While the repeating FRB has been associated with a radio continuum source at high redshift, the nature of radio continuum source is not known. Furthermore, the connection between the repeating FRB (detected with Arecibo) and the apparently non-repeating FRBs (primarily detected with Parkes) is unclear.

\footnotetext{
8 Murase et al. (2016) estimate a lower maximum distance for the FRB, because they assume a Galactic contribution to the dispersion of $200 \mathrm{pc} \mathrm{cm}^{-3}$ While they claim that this is based on the NE2001 Galactic electron density model (Cordes \& Lazio 2002), along this line of sight the electron column density from NE2001 is $70 \mathrm{pc} \mathrm{cm}^{-3}$. An alternative electron density model (Yao et al. 2017) predicts a much larger electron column density along this line of sight of $220 \mathrm{pc} \mathrm{cm}^{-3}$, owing to a large contribution from the Gum nebula.
}

\section{Conclusions}

We present 25 epochs of centimetric imaging observations of the field of FRB 131104 with the ATCA spanning 2.5 years. No radio afterglow is coincident with the $\gamma$-ray event reported by DeLaunay et al. (2016). This can be used to constrain the energetics of the associated cataclysm or suggest, as supported by probabilistic arguments we outline, that the $\gamma$-ray event is unrelated to the FRB or spurious.

We have identified an unusual flaring radio source temporally and spatially coincident with FRB 131104. This source, AT J0642.9-5118, is not spatially coincident with the potential $\gamma$-ray transient Swift J0644.5-5111. AT J0642.9 -5118 is consistent with compact emission components in an AGN, as identified by optical and infrared photometry and spectroscopy. The discovery of further, better-localized FRBs with either radio or $\gamma$-ray flares (or neither) will resolve the uncertainty (or not) in the multiwavelength associations with the enigmatic FRB population.

We thank M. Kasliwal for obtaining and reducing the J-band Magellan data presented in this Letter, H. Vedantham, S. Kulkarni, K. Masui, R. Blandford, and S. Johnston for useful discussions, and S. Ryder and the International Telescope Support Office at the Australian Astronomical Observatory for assistance in coordinating Gemini observations. We thank the group of M. Bailes at the Swinburne University of Technology for making available their real-time FRB detector, without which the rapid follow-up observations of FRB 131104 would not have been possible. We are also grateful for the prompt scheduling of our observations by CSIRO Astronomy and Space Science operations staff. The Australia Telescope Compact Array and Parkes radio telescope are part of the Australia Telescope which is funded by the Commonwealth of Australia for operation as a National Facility managed by the Commonwealth Science and Industrial Research Organization (CSIRO). This Letter is partially based on observations obtained at the Gemini Observatory, which is operated by the Association of Universities for Research in Astronomy, Inc., under a cooperative agreement with the NSF on behalf of the Gemini partnership: the National Science Foundation (United States), the National Research Council (Canada), CONICYT (Chile), Ministerio de Ciencia, Tecnología e Innovación Productiva (Argentina), Ministério da Ciência, Tecnologia e Inovação (Brazil), and, previously, the Department of Industry and Science (Australia). This paper includes data gathered with the $6.5 \mathrm{~m}$ Magellan Telescopes located at Las Campanas Observatory, Chile.

Facilities: Parkes, ATCA, Gemini:South (GMOS), Magellan:Baade (FourStar).

\section{References}

Akiyama, K., \& Johnson, M. D. 2016, ApJL, 824, L3 Bannister, K. W., Stevens, J., Tuntsov, A. V., et al. 2016, Sci, 351, 354 Barthelmy, S. D., Barbier, L. M., Cummings, J. R., et al. 2005, SSRv, 120, 143 Bell, M. E., Huynh, M. T., Hancock, P., et al. 2015, MNRAS, 450, 4221 Cai, Y.-F., Sabancilar, E., Steer, D. A., \& Vachaspati, T. 2012, PhRvD, 86, 043521

Chatterjee, S., Law, C. J., Wharton, R. S., et al. 2017, Natur, 541, 58 Cordes, J. M., \& Lazio, T. J. W. 2002, arXiv:astro-ph/0207156

Cordes, J. M., \& Wasserman, I. 2016, MNRAS, 457, 232

DeLaunay, J. J., Fox, D. B., Murase, K., et al. 2016, ApJL, 831, L1

Dolag, K., Gaensler, B. M., Beck, A. M., \& Beck, M. C. 2015, MNRAS, 451, 4277 
Frail, D. A., Waxman, E., \& Kulkarni, S. R. 2000, ApJ, 537, 191

Gehrels, N., Ramirez-Ruiz, E., \& Fox, D. B. 2009, ARA\&A, 47, 567

Gimeno, G., Roth, K., Chiboucas, K., et al. 2016, Proc. SPIE, 9908, 99082S

Hook, I. M., Jørgensen, I., Allington-Smith, J. R., et al. 2004, PASP, 116, 425

Hovatta, T., Nieppola, E., Tornikoski, M., et al. 2008, A\&A, 485, 51

Johnston, S., Keane, E. F., Bhandari, S., et al. 2017, MNRAS, 465, 2143

Katz, J. I. 2016, ApJ, 826, 226

Keane, E. F., Johnston, S., Bhandari, S., et al. 2016, Natur, 530, 453

Keane, E. F., \& Petroff, E. 2015, MNRAS, 447, 2852

Komossa, S. 2008, RMxAC, 32, 86

Lorimer, D. R., Bailes, M., McLaughlin, M. A., Narkevic, D. J., \& Crawford, F. 2007, Sci, 318, 777

Lyubarsky, Y. 2008, ApJ, 682, 1443

Lyubarsky, Y. 2014, MNRAS, 442, L9

Marcote, B., Paragi, Z., Hessels, J. W. T., et al. 2017, ApJL, 834, L8

Masui, K., Lin, H.-H., Sievers, J., et al. 2015, Natur, 528, 523

Mészáros, P., \& Rees, M. J. 1997, ApJ, 476, 232

Monet, D. G., Levine, S. E., Canzian, B., et al. 2003, AJ, 125, 984

Mooley, K. P., Hallinan, G., Bourke, S., et al. 2016, ApJ, 818, 105

Murase, K., Kashiyama, K., \& Mészáros, P. 2016, MNRAS, 461, 1498

Murase, K., Meszaros, P., \& Fox, D. B. 2017, ApJL, 836, L6

Ofek, E. O., Frail, D. A., Breslauer, B., et al. 2011, ApJ, 740, 65

Osterbrock, D. E. 1978, PhyS, 17, 137

Persson, S. E., Murphy, D. C., Smee, S., et al. 2013, PASP, 125, 654
Polletta, M., Tajer, M., Maraschi, L., et al. 2007, ApJ, 663, 81

Ravi, V., Shannon, R. M., Bailes, M., et al. 2016, Sci, 354, 1249

Ravi, V., Shannon, R. M., \& Jameson, A. 2015, ApJL, 799, L5

Rees, M. J., \& Mészáros, P. 1998, ApJL, 496, L1

Romero, G. E., del Valle, M. V., \& Vieyro, F. L. 2016, PhRvD, 93, 023001

Sault, R. J., Teuben, P. J., \& Wright, M. C. H. 1995, in ASP Conf. Ser. 77,

Astronomical Data Analysis Software and Systems IV, ed. R. A. Shaw,

H. E. Payne, \& J. J. E. Hayes (San Francisco, CA: ASP), 433

Schlafly, E. F., \& Finkbeiner, D. P. 2011, ApJ, 737, 103

Skrutskie, M. F., Cutri, R. M., Stiening, R., et al. 2006, AJ, 131, 1163

Smolčić, V., Ivezić, Ž., Gaćeša, M., et al. 2006, MNRAS, 371, 121

Spitler, L. G., Scholz, P., Hessels, J. W. T., et al. 2016, Natur, 531, 202

Stern, D., Assef, R. J., Benford, D. J., et al. 2012, ApJ, 753, 30

Tendulkar, S. P., Bassa, C. G., Cordes, J. M., et al. 2017, ApJL, 834, L7

Thornton, D., Stappers, B., Bailes, M., et al. 2013, Sci, 341, 53

Vedantham, H. K., Ravi, V., Hallinan, G., \& Shannon, R. M. 2016a, ApJ, 830,75

Vedantham, H. K., Ravi, V., Mooley, K., et al. 2016b, ApJL, 824, L9

Wang, J.-S., Yang, Y.-P., Wu, X.-F., Dai, Z.-G., \& Wang, F.-Y. 2016, ApJL, 822, L7

Williams, P. K. G., \& Berger, E. 2016, ApJL, 821, L22

Wilson, W. E., Ferris, R. H., Axtens, P., et al. 2011, MNRAS, 416, 832

Wright, E. L., Eisenhardt, P. R. M., Mainzer, A. K., et al. 2010, AJ, 140, 1868

Yao, J. M., Manchester, R. N., \& Wang, N. 2017, ApJ, 835, 29 